\title{
Bioavailability and mobility of mercury in selected soil profiles
}

\author{
Szymon Łucjan Różański ${ }^{1}$ Jose Matias Peñas Castejónn ${ }^{2} \cdot$ Gregorio García Fernández $^{2}$
}

Received: 25 September 2015 / Accepted: 19 January 2016/Published online: 6 July 2016

(c) The Author(s) 2016. This article is published with open access at Springerlink.com

\begin{abstract}
To understand the behaviour of mercury in soils and assess the risk of its toxicity, the forms in which the element is found in the environment must be detected. In the occurrence of favourable alkylation conditions (for example in floodplain soils), the content of methyl and ethyl mercury is higher. These $\mathrm{Hg}$ forms in comparison with mineral compounds are toxic for the same organisms in concentrations from 10 to 100 times lower. The research concerned eight selected soil profiles representing six different types of soils (Endogleyic Phaeozem, Endogleyic Fluvisol, Eutric Fluvisol, Haplic Luvisols, Brunic Arenosol, Albic Podzol) placed in croplands. The content of mobile (water soluble), available (DTPA-extractable) and bound with organic matter ( $\mathrm{NaOH}$-extractable) $\mathrm{Hg}$ forms was determined after thermal decomposition using AMA mercury analyser. The mobility as well as availability of mercury in the analysed soils was very low, with average percentage to 0.28 and $2.45 \%$ of the total content of this metal, respectively. It was mainly dependent on texture, the amount of organic matter and soil $\mathrm{pH}$. The percentage of mercury bound with organic matter ranged from 2.34 to $73.70 \%$ of the total content of this metal and was correlated with amount of clay and $\mathrm{Fe}$ oxides. Considering these results, the hazard of migration of this element into deeper horizons of the soil profile and ground water is very low. Moreover, crops from the investigated area are not at risk from mercury contamination.
\end{abstract}

Szymon Łucjan Różański

szymi@utp.edu.pl

1 UTP University of Science and Technology, Bydgoszcz, Poland

2 Technical University of Cartagena UPCT, Cartagena, Spain
Keywords Mercury · Mobility · Bioavailability · Soils · AMA

\section{Introduction}

Mercury possesses unique physicochemical properties when compared to all chemical elements, and the variety of compounds it forms make it both a very difficult and interesting object of study (Fitzgerald 1995; Lindqvist et al. 1991; Schlüter 2000; Schuster 1991). Toxicity of mercury is linked to the form in which it is found in the environment. Organic Hg compounds (methyl mercury and ethyl mercury mostly), in comparison with mineral compounds, are toxic for the same organisms in concentrations from 10 to 100 times lower (Boening 2000; Gochfeld 2003). The form of the highest toxicity and at the same time of the highest bioaccumulation is methyl and dimethyl mercury (Alloway 1995; Gochfeld 2003). This element is very easily bioaccumulated. Plants accumulate relatively small amounts of $\mathrm{Hg}$, much more in animals (especially water animals) and fungi (Boening 2000; Campbell et al. 2003; Kabata-Pendias and Pendias 2000; Tsuchiya et al. 2008). This metal is mainly accumulated in plant roots, from which it is transferred at different rates to the parts above the surface (Boening 2000; Gochfeld 2003).

Mercury undergoes very specific transformations in different ecosystems. It is crucial to observe the content of different forms of this metal in soils. This is correlated with the variety of structure and composition and the dynamics of the soil system. This results in different soil genesis, as well as a variety and variability of environmental conditions and human pressures, both spatially and temporally.

The aim of this study was to assess the influence of selected soil components and physicochemical properties 
in mercury distribution, environmental mobility and potential toxicity of this element in different soil types of croplands.

\section{Materials and methods}

\section{Study area}

The study was carried out in three regions of Poludniowodbaltuckie Lake District, Northern Poland (Fig. 1). The parent materials of studied soils were glacial till deposited on Pomeranian phase of the Vistula glaciation period, fluvioglacial sands formed during the continental ice-sheet recession of the Pomeranian and Holocene phases, and also young fluvial deposits. The mean annual temperature in the study area is $8{ }^{\circ} \mathrm{C}$, and the mean annual precipitation is $500 \mathrm{~mm}$.

\section{Sample collection}

The research material was collected from 45 samples taken from eight selected soil profiles (Fig. 2) representing six types of soils: one profile of Endogleyic Phaeozem, one profile of Endogleyic Fluvisol, one profile of Eutric Fluvisol, three profiles of Haplic Luvisols, one profile of Brunic Arenosol, and one profile of Albic Podzol (IUSS Working Group 2014), formed form parent material of various origins. The analysed soils are arable soils (long-term cultivated) which are used for cultivating plants typical for the region (sugar beet, wheat, rye). Only Albic Podzol is a forest soil located in the area of relatively limited anthropogenic influence. Soil samples were stored in plastic bags and kept in a refrigerator for mercury determination. The coordinates of sampling sites were recorded with GPSprofile (1) $53^{\circ} 12^{\prime} 25.7^{\prime \prime} \mathrm{N} ; \quad 18^{\circ} 09^{\prime} 25.0^{\prime \prime} \mathrm{E}$, profile (2) $53^{\circ} 17^{\prime} 32.6^{\prime \prime} \mathrm{N}$; $18^{\circ} 04^{\prime} 29.9^{\prime \prime} \mathrm{E}$, profile (3) $53^{\circ} 17^{\prime} 31.4^{\prime \prime} \mathrm{N}$; $18^{\circ} 17^{\prime} 17.9^{\prime \prime} \mathrm{E}$, profile (4) $53^{\circ} 14^{\prime} 55.5^{\prime \prime} \mathrm{N} ; 18^{\circ} 16^{\prime} 13.7^{\prime \prime} \mathrm{E}$, profile (5) $53^{\circ} 13^{\prime} 54.4^{\prime \prime} \mathrm{N}$; $18^{\circ} 13^{\prime} 23.5^{\prime \prime} \mathrm{E}$, profile (6) $53^{\circ} 12^{\prime} 50.0^{\prime \prime} \mathrm{N}$; $18^{\circ} 03^{\prime} 49.1^{\prime \prime} \mathrm{E}$, profile (7) $53^{\circ} 15^{\prime} 48.1^{\prime \prime} \mathrm{N}$; $17^{\circ} 57^{\prime} 17.10^{\prime \prime}$, profile (8) $53^{\circ} 11^{\prime} 10.6^{\prime \prime} \mathrm{N} ; 18^{\circ} 05^{\prime} 54.7^{\prime \prime} \mathrm{E}$.

The results presented are a continuation and extension of research on the content of mercury in the soil environment presented by Różański (2009) together with complete data on composition, physicochemical properties, and the total content of mercury.

\section{Chemical analysis}

Each soil sample was air-dried at room temperature until constant mass, crushed, and sieved to separate the $<2 \mathrm{~mm}$ fraction from any gravel or larger detritus.

The following soil properties and components were determined: texture (Bouyoucos hydrometer method), TOC - total organic carbon content (SKALAR analyser), $\mathrm{Fe}_{\text {tot }}$ - - total iron content (ISO 14869-1:2001), $\mathrm{Fe}_{\mathrm{d}}$-content of free iron oxides (Mehra and Jackson 1960), $\mathrm{Fe}_{\mathrm{o}}$ content of amorphous iron oxides (Schwertmann 1964), pH in $\mathrm{H}_{2} \mathrm{O}$ and in $1 \mathrm{M}$ solution of $\mathrm{KCl}$ (ISO 10390:1994), total content of exchangeable hydrogen cations (Kappen method in $1 \mathrm{M} \mathrm{CH}_{3} \mathrm{COONa}$ ), and $\mathrm{CEC}$ - cation exchange capacity (ISO 11260:1994).
Fig. 1 Location of sampling area

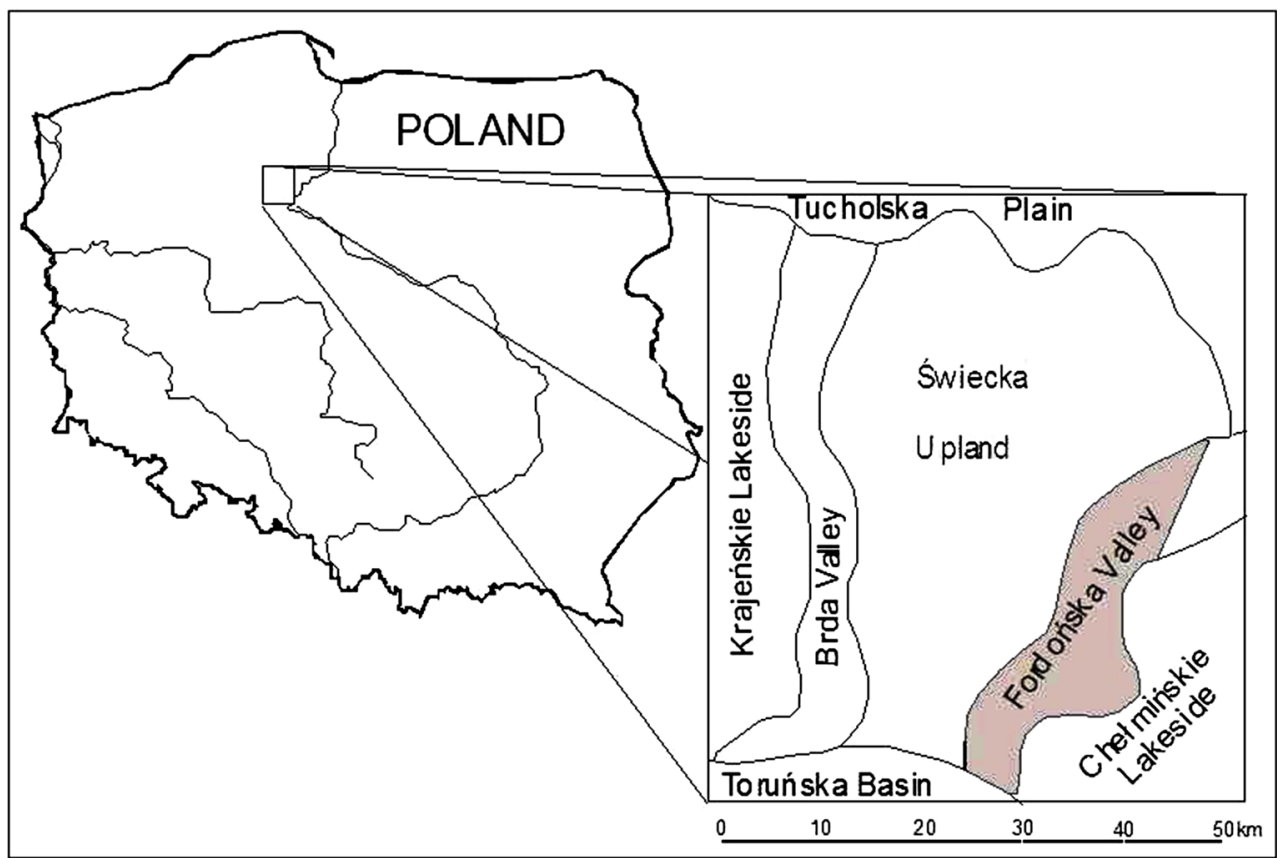



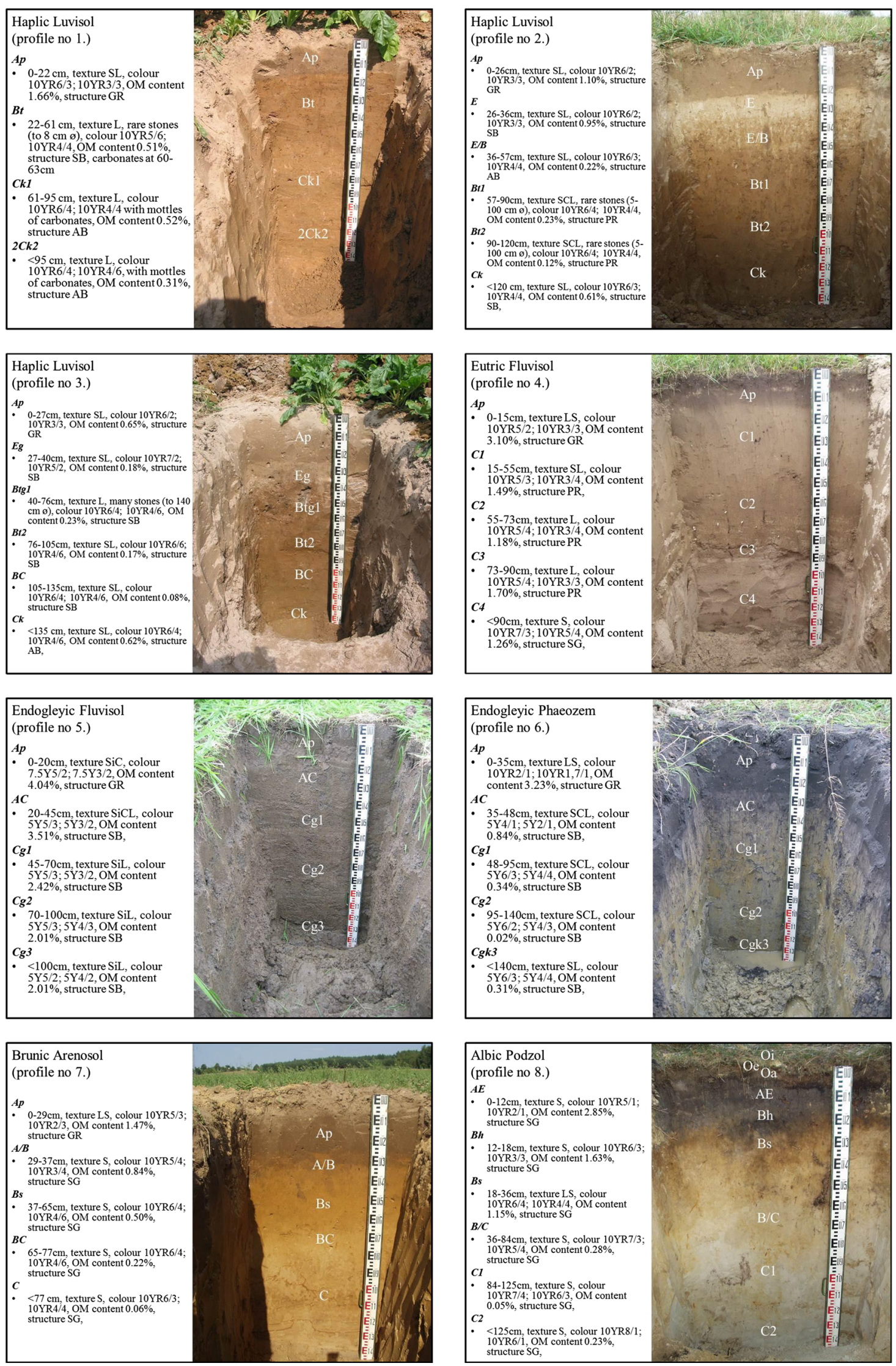

Fig. 2 Morphological description of studied soil profiles 
Total $\mathrm{Hg}$ concentrations and its forms were analysed according to the following procedure. The content of water-soluble, mobile mercury forms $\left(\mathrm{Hg}_{\mathrm{H} 2 \mathrm{O}}\right)$ was extracted using deionized water (Milli-Q Millipore 18.2 M $\mathrm{cm}^{-1}$ at $25^{\circ} \mathrm{C}$ ) in a soil/water ratio $1: 2$ during $16 \mathrm{~h}$. The content of available mercury was extracted by $1 \mathrm{M}$ diethylene triamine pentaacetic acid ( $\left.\mathrm{Hg}_{\mathrm{DTPA}}\right)$ (Lindsay and Norvell 1978) in a soil/DTPA ratio 1:2 during $2 \mathrm{~h}$, and the content of mercury bound to the soil organic matter by $1 \mathrm{M}$ $\mathrm{NaOH}\left(\mathrm{Hg}_{\mathrm{NaOH}}\right)$ in a soil/ $\mathrm{NaOH}$ ratio 1:20 during $2 \mathrm{~h}$ (Schnitzer and Khan 1978). Mercury was determined on advanced mercury analyser, the AMA-254 in solid $\left(\mathrm{Hg}_{\text {tot }}\right)$ and liquid $\left(\mathrm{Hg}_{\mathrm{H} 2 \mathrm{O}}, \mathrm{Hg}_{\mathrm{DTPA}}\right.$, and $\left.\mathrm{Hg}_{\mathrm{NaOH}}\right)$ samples. The system allows for the thermal heating $\left(750{ }^{\circ} \mathrm{C}\right)$ of the sample in flow of oxygen to decompose the sample in quartz tube and release mercury, which is concentrated in a gold amalgamator. The mercury vapours are measured at $253.65 \mathrm{~nm}$ by a silicon diode UV detector. The AMA-254 has a 0.01-ng $\mathrm{Hg}$ detection limit, a working range from 0.05 to $600 \mathrm{ng} \mathrm{Hg}$, reproducibility smaller than $1.5 \%$. $\mathrm{Hg}_{\text {tot }}$ was determined in solid $300 \mathrm{mg}$ samples in five replications, and $\mathrm{Hg}_{\mathrm{H} 2 \mathrm{O}}, \mathrm{Hg}_{\mathrm{DTPA}}$, and $\mathrm{Hg}_{\mathrm{NaOH}}$ were determined in liquid samples in three replications the volume from 1000 to $1500 \mu \mathrm{L}$, depending on the mercury concentration in the extract.

All the results of the mercury content in soil samples were verified using certified material (reference soil sample TILL-3 and SO-4, Canada Center for Mineral and Energy Technology).

\section{Results and discussion}

\section{Content of water-soluble mercury}

It is assumed that the water-soluble mercury compounds are mainly salts-e.g. $\mathrm{HgCl}_{2}, \mathrm{HgBr}_{2}, \mathrm{Hg}(\mathrm{CN})_{2}, \mathrm{Hg}_{2}$ $\left(\mathrm{C}_{2} \mathrm{H}_{3} \mathrm{O}_{2}\right)_{2}$ (Barnett and Turner 2001; Han et al. 2003; Schroeder and Munthe 1998), and also low-molecularweight organic $\mathrm{Hg}$ compounds (Wallschläger et al. 1998a). It should, however, be noted that the solubility of mercury compounds depends to a great extent on the composition and properties of the soil solution. For example, cinnabar ( $\mathrm{HgS})$, which is sparingly soluble, may dissolve in solutions containing dissolved organic matter (fulvic acids) or compounds with thiol ligands (Jacobson et al. 2005). The content of soluble fractions in soils does not usually exceed $4 \%$ of the total organic matter content (Henderson et al. 1998).

The content of mobile, water-soluble mercury forms was very low in the examined soils and ranged between 0 and $0.82 \mu \mathrm{g} \cdot \mathrm{kg}^{-1}$ (Table 2). The percentage of $\mathrm{Hg}_{\mathrm{H} 2 \mathrm{O}}$ in the total content of this metal was on average $0.28 \%$. Low share of readily soluble $\mathrm{Hg}$ compounds indicates that their migration into the deeper soil horizons is low. The highest content of $\mathrm{Hg}_{\mathrm{H} 2 \mathrm{O}}$ was found in surface horizons, especially organic horizons of Albic Podzol (profile 8). Slightly different results were found for the polluted soils of Europe, where the highest content of mobile mercury forms was found in subsurface horizons (below $20 \mathrm{~cm}$ ). This was linked with $\mathrm{Hg}$ being washed together with humus acids from surface horizons to subsurface horizons, where these were sorbed by mineral components of soil sorption complex (Biester et al. 2002a, b).

Forest soils of northern Poland were characterized by higher content of $\mathrm{Hg}$ than the content found in this research (Malczyk 2000), while in the mercury-polluted soils of Great Britain, the concentration of $\mathrm{Hg}_{\mathrm{H} 2 \mathrm{O}}$ was so low that it did not exceed the detection level in the analytic method used (Panyametheekul 2004). In the soils of central Spain, the content of $\mathrm{Hg}_{\mathrm{H} 2 \mathrm{O}}$ did not exceed the level of $0.025 \mu \mathrm{g} \cdot \mathrm{kg}^{-1}$ (using both the AMA and ICP-MS method) (Sánchez et al. 2005).

The content of readily soluble mercury compounds was positively correlated with the content of organic carbon $(r=0.51 ; p<0.05$, Table 3$)$. The highest content of $\mathrm{Hg}_{\mathrm{H} 2 \mathrm{O}}$ was determined in surface horizons of the analysed soils, rich in organic matter (except profile 3 and 7 , Tables 1, 2). The greatest percentage of water-soluble forms in the total content of $\mathrm{Hg}$ was found in horizons with considerably low content of organic matter and poor in clay fraction (especially in E horizons of Haplic Luvisols, profiles 2 and 3). Such results indicate considerable influence of both organic matter and clay fraction on the content of $\mathrm{Hg}_{\mathrm{H} 2 \mathrm{O}}$.

These results indicate that the amount of readily soluble $\mathrm{Hg}$ forms was relatively low in relatively fine-textured soil, rich in clay minerals (profile 5). When comparing the content of $\mathrm{Hg}_{\mathrm{H} 2 \mathrm{O}}$ in surface horizons in both analysed Fluvisols (profiles 4 and 5), it could be concluded that the percentage of these $\mathrm{Hg}$ forms, despite comparable content of organic carbon, and considerably high total content of mercury in fine-textured Endogleyic Fluvisol (profile 5), was significantly higher in coarse-textured Eutric Fluvisol (profile 4, Tables 1, 2). Therefore, it seems that clay fraction was very influential for binding mercury in these soils. Moreover, the content of the analysed Fluvisols suggests that in soils enriched in humus and clay minerals, as a result of adsorption of positively charged mercury cations on the negatively charged surface of humus compounds and clay minerals, sparingly soluble in water complexes $\mathrm{Hg}$-humus-clay minerals may be formed. Mercury is hence bound to the solid phase of the soil. This could account for a relatively high content of $\mathrm{Hg}_{\mathrm{H} 2 \mathrm{O}}$ in organic horizons $(\mathrm{Oi}$, $O e$, and $\mathrm{Oa}$ ) of the Albic Podzol (profile 8), in which no clay minerals were found. The above hypothesis stating the 
Table 1 Determined components and properties of studied soil profiles (Różański 2009)

\begin{tabular}{|c|c|c|c|c|c|c|c|c|c|c|c|}
\hline $\begin{array}{l}\text { Profile } \\
\text { no }\end{array}$ & Horizon & $\begin{array}{l}\text { Depth } \\
(\mathrm{cm})\end{array}$ & $\begin{array}{l}\text { Clay } \\
(\%)\end{array}$ & $\begin{array}{l}\text { TOC } \\
\left(\mathrm{g} \mathrm{kg}^{-1}\right)\end{array}$ & $\begin{array}{l}\mathrm{pH} \\
\mathrm{H}_{2} \mathrm{O}\end{array}$ & $\begin{array}{l}\mathrm{pH} \\
\mathrm{KCl}\end{array}$ & $\begin{array}{l}\mathrm{H}^{+} \\
\left(\mathrm{mmol} \mathrm{kg}^{-1}\right)\end{array}$ & $\begin{array}{l}\text { CEC } \\
\left(\mathrm{mmol} \mathrm{kg}^{-1}\right)\end{array}$ & $\begin{array}{l}\mathrm{Fe}_{\text {tot }} \\
\left(\mathrm{g} \mathrm{kg}^{-1}\right)\end{array}$ & $\begin{array}{l}\mathrm{Fe}_{\mathrm{d}} \\
\left(\mathrm{g} \mathrm{kg}^{-1}\right)\end{array}$ & $\begin{array}{l}\mathrm{Fe}_{\mathrm{o}} \\
\left(\mathrm{g} \mathrm{kg}^{-1}\right)\end{array}$ \\
\hline \multicolumn{12}{|c|}{ Haplic Luvisol } \\
\hline \multirow[t]{4}{*}{1} & Ap & $0-22$ & 15 & 9.7 & 6.5 & 5.9 & 5.2 & 68.2 & 12.93 & 4.3 & 1.4 \\
\hline & $\mathrm{Bt}$ & $22-61$ & 22 & 3.0 & 6.3 & 4.9 & 5.0 & 116.0 & 24.55 & 8.0 & 1.7 \\
\hline & Ck1 & $61-95$ & 17 & 1.2 & 8.0 & 7.3 & 0.8 & 100.7 & 17.53 & 4.5 & 0.7 \\
\hline & $2 \mathrm{Ck} 2$ & $<95$ & 26 & 1.0 & 8.0 & 7.3 & 0.8 & 81.5 & 20.02 & 6.0 & 0.6 \\
\hline \multicolumn{12}{|c|}{ Haplic Luvisol } \\
\hline \multirow[t]{6}{*}{2} & Ap & $0-26$ & 7 & 6.4 & 5.7 & 4.7 & 13.5 & 43.9 & 7.91 & 2.1 & 0.8 \\
\hline & $\mathrm{E}$ & $26-36$ & 5 & 5.5 & 5.8 & 4.6 & 6.9 & 39.1 & 8.58 & 1.8 & 0.5 \\
\hline & $\mathrm{E} / \mathrm{B}$ & $36-57$ & 18 & 1.3 & 6.3 & 4.9 & 4.8 & 82.6 & 18.19 & 4.5 & 1.0 \\
\hline & Bt1 & $57-90$ & 22 & 1.4 & 6.7 & 5.2 & 4.5 & 112.1 & 25.43 & 6.9 & 1.3 \\
\hline & $\mathrm{Bt} 2$ & $90-120$ & 23 & 0.7 & 7.1 & 5.9 & 3.4 & 124.5 & 22.78 & 6.2 & 1.0 \\
\hline & $\mathrm{Ck}$ & $<120$ & 17 & 0.7 & 8.0 & 7.3 & 0.7 & 103.5 & 18.07 & 4.2 & 0.5 \\
\hline \multicolumn{12}{|c|}{ Haplic Luvisol } \\
\hline \multirow[t]{6}{*}{3} & Ap & $0-27$ & 4 & 3.8 & 6.8 & 6.6 & 1.8 & 28.0 & 7.17 & 2.1 & 0.9 \\
\hline & $\mathrm{Eg}$ & $27-40$ & 1 & 1.1 & 6.6 & 5.6 & 2.8 & 25.0 & 7.66 & 1.7 & 0.8 \\
\hline & Btg1 & $40-76$ & 20 & 1.4 & 6.9 & 5.3 & 5.4 & 145.4 & 29.03 & 7.6 & 2.6 \\
\hline & $\mathrm{Bt} 2$ & $76-105$ & 18 & 1.0 & 6.5 & 5.0 & 4.5 & 107.0 & 21.88 & 6.1 & 1.4 \\
\hline & $\mathrm{BC}$ & $105-135$ & 16 & 0.5 & 7.3 & 6.5 & 2.2 & 93.2 & 19.20 & 5.3 & 1.2 \\
\hline & $\mathrm{Ck}$ & $<135$ & 13 & 0.4 & 8.0 & 7.4 & 0.6 & 84.6 & 16.45 & 4.6 & 0.4 \\
\hline \multicolumn{12}{|c|}{ Eutric Fluvisol } \\
\hline \multirow[t]{5}{*}{4} & Ap & $0-15$ & 7 & 18.0 & 7.0 & 6.7 & 3.2 & 93.3 & 9.65 & 3.7 & 2.0 \\
\hline & $\mathrm{C} 1$ & $15-55$ & 10 & 8.7 & 7.4 & 7.2 & 1.7 & 98.6 & 12.47 & 4.4 & 2.5 \\
\hline & $\mathrm{C} 2$ & $55-73$ & 15 & 6.9 & 7.6 & 7.2 & 1.6 & 153.1 & 18.07 & 7.0 & 3.8 \\
\hline & $\mathrm{C} 3$ & $73-90$ & 13 & 9.9 & 7.7 & 7.3 & 1.4 & 139.7 & 16.72 & 6.1 & 3.4 \\
\hline & $\mathrm{C} 4$ & $<90$ & 2 & 7.3 & 7.8 & 7.4 & 2.0 & 19.6 & 2.34 & 0.9 & 0.4 \\
\hline \multicolumn{12}{|c|}{ Endogleyic Fluvisol } \\
\hline \multirow[t]{5}{*}{5} & Ap & $0-20$ & 42 & 23.5 & 7.5 & 7.1 & 2.3 & 317.2 & 39.59 & 18.9 & 8.1 \\
\hline & $\mathrm{AC}$ & $20-45$ & 29 & 20.4 & 7.6 & 7.0 & 1.8 & 252.1 & 33.65 & 15.1 & 6.4 \\
\hline & $\mathrm{Cg} 1$ & $45-70$ & 26 & 14.1 & 7.7 & 7.1 & 1.6 & 236.7 & 30.22 & 13.5 & 5.9 \\
\hline & $\mathrm{Cg} 2$ & $70-100$ & 21 & 11.7 & 7.8 & 7.2 & 1.9 & 198.6 & 24.03 & 10.6 & 4.7 \\
\hline & $\mathrm{Cg} 3$ & $<100$ & 20 & 11.7 & 7.8 & 7.2 & 1.6 & 196.9 & 24.48 & 10.1 & 3.4 \\
\hline \multicolumn{12}{|c|}{ Endogleyic Phaeozem } \\
\hline \multirow[t]{5}{*}{6} & Ap & $0-35$ & 8 & 18.8 & 6.2 & 5.8 & 7.5 & 138.3 & 7.17 & 1.8 & 0.7 \\
\hline & $\mathrm{AC}$ & $35-48$ & 23 & 4.9 & 7.0 & 5.8 & 4.2 & 150.9 & 15.63 & 2.3 & 0.3 \\
\hline & $\mathrm{Cg} 1$ & $48-95$ & 22 & 2.0 & 7.5 & 6.4 & 2.1 & 132.1 & 23.17 & 10.5 & 0.5 \\
\hline & $\mathrm{Cg} 2$ & $95-140$ & 20 & 0.1 & 7.6 & 6.5 & 2.3 & 107.0 & 18.76 & 5.3 & 0.4 \\
\hline & $\mathrm{Cgk} 3$ & $<140$ & 19 & 0.3 & 7.9 & 7.3 & 1.4 & 106.8 & 18.33 & 5.3 & 0.6 \\
\hline \multicolumn{12}{|c|}{ Brunic Arenosol } \\
\hline \multirow[t]{5}{*}{7} & Ap & $0-29$ & 3 & 8.5 & 4.8 & 4.1 & 20.9 & 30.3 & 5.90 & 2.3 & 1.1 \\
\hline & $\mathrm{A} / \mathrm{B}$ & $29-37$ & 4 & 4.9 & 5.3 & 4.5 & 12.0 & 18.6 & 5.21 & 2.2 & 1.6 \\
\hline & Bs & $37-65$ & 5 & 2.9 & 5.7 & 4.8 & 13.4 & 25.3 & 5.15 & 2.3 & 1.4 \\
\hline & $\mathrm{BC}$ & $65-77$ & 2 & 1.3 & 6.3 & 5.1 & 7.3 & 20.2 & 5.12 & 1.4 & 0.6 \\
\hline & $\mathrm{C}$ & $<77$ & 3 & 0.4 & 6.6 & 5.2 & 6.1 & 21.7 & 3.94 & 1.1 & 0.4 \\
\hline
\end{tabular}


Table 1 continued

\begin{tabular}{|c|c|c|c|c|c|c|c|c|c|c|c|}
\hline $\begin{array}{l}\text { Profile } \\
\text { no }\end{array}$ & Horizon & $\begin{array}{l}\text { Depth } \\
(\mathrm{cm})\end{array}$ & $\begin{array}{l}\text { Clay } \\
(\%)\end{array}$ & $\begin{array}{l}\text { TOC } \\
\left(\mathrm{g} \mathrm{kg}^{-1}\right)\end{array}$ & $\begin{array}{l}\mathrm{pH} \\
\mathrm{H}_{2} \mathrm{O}\end{array}$ & $\begin{array}{l}\mathrm{pH} \\
\mathrm{KCl}\end{array}$ & $\begin{array}{l}\mathrm{H}^{+} \\
\left(\mathrm{mmol} \mathrm{kg}^{-1}\right)\end{array}$ & $\begin{array}{l}\text { CEC } \\
\left(\mathrm{mmol} \mathrm{kg}^{-1}\right)\end{array}$ & $\begin{array}{l}\mathrm{Fe}_{\text {tot. }} \cdot \\
\left(\mathrm{g} \mathrm{kg}^{-1}\right)\end{array}$ & $\begin{array}{l}\mathrm{Fe}_{\mathrm{d}} \\
\left(\mathrm{g} \mathrm{kg}^{-1}\right)\end{array}$ & $\begin{array}{l}\mathrm{Fe}_{\mathrm{o}} \\
\left(\mathrm{g} \mathrm{kg}^{-1}\right)\end{array}$ \\
\hline \multicolumn{12}{|c|}{ Albic Podzol } \\
\hline \multirow[t]{9}{*}{8} & Oi & $10-9$ & - & 448.2 & 4.7 & 4.3 & 104.3 & 852.5 & 1.94 & 1.0 & 0.4 \\
\hline & $\mathrm{Oe}$ & $9-3$ & - & 485.2 & 4.3 & 3.7 & 189.0 & 816.9 & 5.17 & 2.7 & 1.4 \\
\hline & $\mathrm{Oa}$ & $3-0$ & - & 216.9 & 4.2 & 3.5 & 144.0 & 515.3 & 5.58 & 3.5 & 1.8 \\
\hline & $\mathrm{AE}$ & $0-12$ & 5 & 16.6 & 4.4 & 3.9 & 48.8 & 55.1 & 2.82 & 1.4 & 0.9 \\
\hline & $\mathrm{Bh}$ & $12-18$ & 6 & 9.5 & 4.7 & 4.3 & 25.2 & 27.4 & 2.82 & 1.5 & 1.2 \\
\hline & Bs & $18-36$ & 8 & 6.7 & 4.6 & 4.5 & 18.3 & 19.8 & 3.10 & 1.7 & 1.4 \\
\hline & $\mathrm{B} / \mathrm{C}$ & $36-84$ & 4 & 1.6 & 4.8 & 4.6 & 12.3 & 14.4 & 2.59 & 0.8 & 0.4 \\
\hline & $\mathrm{C} 1$ & $84-125$ & 4 & 0.3 & 4.8 & 4.7 & 7.5 & 13.0 & 2.99 & 0.8 & 0.4 \\
\hline & $\mathrm{C} 2$ & $<125$ & 3 & 1.4 & 5.1 & 4.7 & 6.7 & 14.0 & 2.77 & 0.4 & 0.1 \\
\hline
\end{tabular}

role of clay minerals in binding mercury in soil has also been confirmed by other authors (Biester et al. 2002a; Boszke et al. 2004; Inácio et al. 1998).

\section{Content of bioavailable mercury}

The content of DTPA-extractable metals in soils are considered as fractions available for plants (Kabata-Pendias and Pendias 2000; Lindsay and Norvell 1978). The percentage of $\mathrm{Hg}_{\text {DTPA }}$ in the total content of mercury was low and ranged between 0.01 and $13.26 \%$ (2.45\% on average, Table 2). A comparable participation of available forms was found by Barnett and Turner (2001) in soils polluted by this metal $(0.3-14 \%, 3.2 \%$ on average). However, these cited authors found that higher available mercury values were in the subsurface horizons, which contrasts with the results of this study since in this case the highest values of available mercury appeared mostly associated with both surface (profiles 1, 2, 4, 7 and 8) and subsurface (usually the parent material horizons-profiles, 3, 5, and 6) horizons.

The concentration of bioavailable forms of $\mathrm{Hg}$ in the examined soils ranged between 0.09 and $2.20 \mu \mathrm{g} \mathrm{kg}^{-1}$ in mineral horizons $\left(0.39 \mu \mathrm{g} \mathrm{kg}^{-1}\right.$ on average $)$ and from 1.21 to $6.83 \mu \mathrm{g} \mathrm{kg}^{-1}$ in organic horizons $\left(4.50 \mu \mathrm{g} \mathrm{kg}^{-1}\right.$ on average, Table 2). The profile distribution for $\mathrm{Hg}_{\mathrm{DTPA}}$ was not homogeneous. No statistically significant correlation was determined between the content of this mercury fraction and total content of the metal. Significant correlations were stated for $\mathrm{Hg}_{\text {DTPA }}$ with acidity $\left(\mathrm{pH}\right.$ in $\mathrm{H}_{2} \mathrm{O}$ and in $\mathrm{KCl}$ ), total content of exchangeable $\mathrm{H}^{+}$cations, total content of $\mathrm{Fe}$ and content of the clay $(r=-0.72$, $r=-0.58, r=0.81, r=-0.59, r=-0.49 ; p<0.05$, respectively, Table 3 ).

In the study of the content of available, DTPA-extractable, mercury forms in soils with acid reaction (Brunic Arenosol and Albic Podzol, profiles 7 and 8, Table 1), the content of these forms was higher in comparison with soils with neutral reaction (Fluvisols and Endogleyic Phaeozem, profiles 4, 5, and 6). Higher content of $\mathrm{Hg}_{\mathrm{DTPA}}$ in these acidic soils may be the result of the release of mercury from Fe and Al complexes (Schlüter 1997). Regarding this, it must be taken into account that the acid reaction increases the content of soluble, low-molecular-weight fulvic acids, mainly responsible for binding of $\mathrm{Hg}$ in the soil solution (Biester et al. 2002a; Wallschläger et al. 1998a). Another important fact in this regard is the important role of $\mathrm{pH}$ fluctuations, which influence the methyl mercury sorption more than $\mathrm{Hg}^{2+}$ sorption (Boszke et al. 2003).

A relatively low content of DTPA-extractable mercury should be noted in Fluvisols and Bt horizons of Luvisols (profiles 1-3). These soils were characterized by considerably high content of clay and amorphous Fe oxides in comparison with other examined samples (Table 1). High content of clay fraction and $\mathrm{Fe}_{\mathrm{o}}$, which forms soil sorption complex, leads to binding of mercury by the solid phase of the soil (Boszke et al. 2003; Dreher and Follmer 2004). This fact does not limit mercury bioavailability when soil shows low amount of organic matter (Biester et al. 2002a; Wang et al. 1997). The process of mercury binding by clay minerals has been observed in soils containing a minimum of $15 \%$ of clay (Wang et al. 1997).

Toxicity of mercury depends on formation of compounds with alkyl groups (mostly methyl group), and therefore alkylation is a very important process from this point of view. It usually takes place in alluvial soils, in which this process, due to high content of organic matter, high level of ground water and seasonal floods, may occur quite fast (Montgomery et al. 2000). Such a direction in transformation of mercury compounds may have influenced the distribution of DTPA-extractable mercury forms in the profiles of Fluvisols and Endogleyic Phaeozem (profiles 4, 5, and 6). In horizons of gleyic characteristics 
Table 2 Total mercury content and its forms

\begin{tabular}{|c|c|c|c|c|c|c|c|c|c|}
\hline $\begin{array}{l}\text { Profile } \\
\text { no }\end{array}$ & Horizon & $\begin{array}{l}\text { Depth } \\
\text { (cm) }\end{array}$ & $\begin{array}{l}\mathrm{Hg}_{\text {tot }} \cdot * \\
\left(\mu \mathrm{gg}^{-1}\right)\end{array}$ & $\begin{array}{l}\mathrm{Hg}_{\mathrm{H} 2 \mathrm{O}} \\
\left(\mu \mathrm{g} \mathrm{kg}^{-1}\right)\end{array}$ & $\begin{array}{l}\mathrm{Hg}_{\text {DTPA }} \\
\left(\mu \mathrm{g} \mathrm{kg}^{-1}\right)\end{array}$ & $\begin{array}{l}\mathrm{Hg}_{\mathrm{NaOH}} \\
\left(\mu \mathrm{g} \mathrm{kg}^{-1}\right)\end{array}$ & $\begin{array}{l}\mathrm{Hg}_{\mathrm{H} 2 \mathrm{O}} \\
(\%)\end{array}$ & $\begin{array}{l}\mathrm{Hg}_{\mathrm{DTPA}} \\
(\%)\end{array}$ & $\begin{array}{l}\mathrm{Hg}_{\mathrm{NaOH}} \\
(\%)\end{array}$ \\
\hline \multicolumn{10}{|c|}{ Haplic Luvisol } \\
\hline \multirow[t]{4}{*}{1} & Ap & $0-22$ & 48.62 & 0.23 & 0.27 & 35.83 & 0.47 & 0.56 & 73.70 \\
\hline & $\mathrm{Bt}$ & $22-61$ & 36.26 & 0.06 & 0.13 & 16.68 & 0.17 & 0.36 & 46.01 \\
\hline & Ck1 & $61-95$ & 19.07 & 0.02 & 0.15 & 8.99 & 0.10 & 0.78 & 47.13 \\
\hline & $2 \mathrm{Ck} 2$ & $<95$ & 19.50 & 0.05 & 0.12 & 7.86 & 0.26 & 0.64 & 40.31 \\
\hline \multicolumn{10}{|c|}{ Haplic Luvisol } \\
\hline \multirow[t]{6}{*}{2} & Ap & $0-26$ & 26.93 & 0.13 & 0.61 & 15.62 & 0.48 & 2.26 & 57.99 \\
\hline & $\mathrm{E}$ & $26-36$ & 16.01 & 0.08 & 0.28 & 6.51 & 0.50 & 1.76 & 40.68 \\
\hline & $\mathrm{E} / \mathrm{B}$ & $36-57$ & 21.60 & 0.04 & 0.21 & 9.06 & 0.18 & 0.97 & 41.96 \\
\hline & Bt1 & $57-90$ & 22.15 & 0.04 & 0.10 & 7.32 & 0.18 & 0.47 & 33.04 \\
\hline & Bt2 & $90-120$ & 33.04 & 0.01 & 0.09 & 8.77 & 0.03 & 0.26 & 26.54 \\
\hline & $\mathrm{Ck}$ & $<120$ & 17.67 & 0.01 & 0.11 & 4.64 & 0.06 & 0.62 & 26.26 \\
\hline \multicolumn{10}{|c|}{ Haplic Luvisol } \\
\hline \multirow[t]{6}{*}{3} & Ap & $0-27$ & 26.61 & 0.09 & 0.30 & 14.01 & 0.34 & 1.13 & 52.66 \\
\hline & $\mathrm{Eg}$ & $27-40$ & 14.19 & 0.10 & 0.34 & 4.90 & 0.70 & 2.36 & 34.53 \\
\hline & Btg1 & $40-76$ & 36.85 & 0.08 & 0.08 & 10.79 & 0.22 & 0.22 & 29.28 \\
\hline & Bt2 & $76-105$ & 29.38 & 0.09 & 0.09 & 6.68 & 0.31 & 0.31 & 22.74 \\
\hline & $\mathrm{BC}$ & $105-135$ & 31.30 & 0.02 & 0.09 & 7.73 & 0.06 & 0.29 & 24.71 \\
\hline & $\mathrm{Ck}$ & $<135$ & 17.08 & 0.02 & 0.16 & 4.02 & 0.12 & 0.94 & 23.52 \\
\hline \multicolumn{10}{|c|}{ Eutric Fluvisol } \\
\hline \multirow[t]{5}{*}{4} & Ap & $0-15$ & 142.90 & 0.42 & 0.49 & 76.00 & 0.29 & 0.35 & 53.18 \\
\hline & $\mathrm{C} 1$ & $15-55$ & 80.60 & 0.08 & 0.12 & 43.97 & 0.10 & 0.15 & 54.56 \\
\hline & $\mathrm{C} 2$ & $55-73$ & 88.93 & 0.04 & 0.12 & 48.36 & 0.04 & 0.14 & 54.38 \\
\hline & $\mathrm{C} 3$ & $73-90$ & 71.90 & 0.03 & 0.14 & 35.23 & 0.04 & 0.19 & 49.00 \\
\hline & $\mathrm{C} 4$ & $<90$ & 3.73 & 0.04 & 0.38 & 1.18 & 1.07 & 10.19 & 31.59 \\
\hline \multicolumn{10}{|c|}{ Endogleyic Fluvisol } \\
\hline \multirow[t]{5}{*}{5} & Ap & $0-20$ & 1438.00 & 0.18 & 0.20 & 33.70 & 0.01 & 0.01 & 2.34 \\
\hline & $\mathrm{AC}$ & $20-45$ & 280.50 & 0.05 & 0.21 & 113.82 & 0.02 & 0.07 & 40.58 \\
\hline & $\mathrm{Cg} 1$ & $45-70$ & 384.70 & 0.08 & 0.19 & 159.37 & 0.02 & 0.05 & 41.43 \\
\hline & $\mathrm{Cg} 2$ & $70-100$ & 292.00 & 0.04 & 0.24 & 142.49 & 0.01 & 0.08 & 48.80 \\
\hline & $\mathrm{Cg} 3$ & $<100$ & 238.40 & 0.04 & 0.24 & 96.70 & 0.02 & 0.10 & 40.56 \\
\hline \multicolumn{10}{|c|}{ Endogleyic Phaeozem } \\
\hline \multirow[t]{5}{*}{6} & Ap & $0-35$ & 36.66 & 0.20 & 0.39 & 23.46 & 0.54 & 1.07 & 63.99 \\
\hline & $\mathrm{AC}$ & $35-48$ & 21.66 & 0.09 & 0.28 & 8.83 & 0.41 & 1.29 & 40.77 \\
\hline & $\mathrm{Cg} 1$ & $48-95$ & 23.26 & $b d l$ & 0.31 & 9.46 & $b d l$ & 1.32 & 40.65 \\
\hline & $\mathrm{Cg} 2$ & $95-140$ & 17.60 & 0.01 & 0.44 & 6.43 & 0.06 & 2.52 & 36.51 \\
\hline & Cgk3 & $<140$ & 18.13 & 0.02 & 0.32 & 3.82 & 0.11 & 1.74 & 21.07 \\
\hline \multicolumn{10}{|c|}{ Brunic Arenosol } \\
\hline \multirow[t]{5}{*}{7} & Ap & $0-29$ & 24.64 & 0.09 & 2.20 & 14.36 & 0.37 & 8.92 & 58.26 \\
\hline & $\mathrm{A} / \mathrm{B}$ & $29-37$ & 22.20 & 0.11 & 0.53 & 13.03 & 0.50 & 2.40 & 58.71 \\
\hline & Bs & $37-65$ & 21.71 & 0.05 & 0.36 & 11.55 & 0.23 & 1.64 & 53.19 \\
\hline & BC & $65-77$ & 8.95 & 0.09 & 0.29 & 2.75 & 1.00 & 3.21 & 30.68 \\
\hline & $\mathrm{C}$ & $<77$ & 6.98 & 0.10 & 0.28 & 0.78 & 1.43 & 3.96 & 11.22 \\
\hline
\end{tabular}


Table 2 continued

\begin{tabular}{|c|c|c|c|c|c|c|c|c|c|}
\hline $\begin{array}{l}\text { Profile } \\
\text { no }\end{array}$ & Horizon & $\begin{array}{l}\text { Depth } \\
(\mathrm{cm})\end{array}$ & $\begin{array}{l}\mathrm{Hg}_{\text {tot }} * \\
\left(\mu \mathrm{g} \mathrm{kg}^{-1}\right)\end{array}$ & $\begin{array}{l}\mathrm{Hg}_{\mathrm{H} 2 \mathrm{O}} \\
\left(\mu \mathrm{g} \mathrm{kg}^{-1}\right)\end{array}$ & $\begin{array}{l}\mathrm{Hg}_{\text {DTPA }} \\
\left(\mu \mathrm{g} \mathrm{kg}^{-1}\right)\end{array}$ & $\begin{array}{l}\mathrm{Hg}_{\mathrm{NaOH}} \\
\left(\mu \mathrm{g} \mathrm{kg}^{-1}\right)\end{array}$ & $\begin{array}{l}\mathrm{Hg}_{\mathrm{H} 2 \mathrm{O}} \\
(\%)\end{array}$ & $\begin{array}{l}\mathrm{Hg}_{\text {DTPA }} \\
(\%)\end{array}$ & $\begin{array}{l}\mathrm{Hg}_{\mathrm{NaOH}} \\
(\%)\end{array}$ \\
\hline \multicolumn{10}{|c|}{ Albic Podzol } \\
\hline \multirow[t]{9}{*}{8} & Oi & $10-9$ & 126.50 & 0.59 & 5.45 & 58.40 & 0.46 & 4.31 & 46.17 \\
\hline & Oe & $9-3$ & 266.70 & 0.38 & 1.21 & 85.86 & 0.14 & 0.46 & 32.19 \\
\hline & $\mathrm{Oa}$ & $3-0$ & 322.00 & 0.82 & 6.83 & 76.06 & 0.25 & 2.12 & 23.62 \\
\hline & $\mathrm{AE}$ & $0-12$ & 21.12 & 0.09 & 1.46 & 9.93 & 0.43 & 6.93 & 47.00 \\
\hline & $\mathrm{Bh}$ & $12-18$ & 19.56 & 0.01 & 0.99 & 8.82 & 0.05 & 5.08 & 45.11 \\
\hline & Bs & $18-36$ & 23.11 & 0.02 & 0.73 & 12.57 & 0.09 & 3.14 & 54.38 \\
\hline & $\mathrm{B} / \mathrm{C}$ & $36-84$ & 7.40 & $b d l$ & 0.98 & 1.81 & $b d l$ & 13.26 & 24.52 \\
\hline & $\mathrm{C} 1$ & $84-125$ & 6.88 & $b d l$ & 0.74 & 1.40 & $b d l$ & 10.79 & 20.39 \\
\hline & $\mathrm{C} 2$ & $<125$ & 5.56 & 0.01 & 0.61 & 0.85 & 0.18 & 10.98 & 15.34 \\
\hline
\end{tabular}

$b d l$ below detection limit

* Różański (2009)

Table 3 Statistically significant relationship between mercury forms and main soil properties

\begin{tabular}{lcccccccccc}
\hline $\mathrm{Hg}$ form & $\mathrm{Hg}_{\text {tot }}$. & $\mathrm{TOC}$ & $\mathrm{pH} \mathrm{H} \mathrm{H}_{2} \mathrm{O}$ & $\mathrm{pH} \mathrm{KCl}$ & $\mathrm{H}^{+}$ & $\mathrm{Fe}_{\text {tot }} \cdot$ & $\mathrm{Fe}_{\mathrm{d}}$ & $\mathrm{Fe}_{\mathrm{o}}$ & Clay & $\mathrm{CEC}$ \\
\hline $\mathrm{Hg}_{\mathrm{H} 2 \mathrm{O}}$ & & 0.51 & & & & & & & & \\
$\mathrm{Hg}_{\mathrm{DTPA}}$ & & & -0.72 & -0.58 & 0.81 & -0.59 & & & & -0.49 \\
$\mathrm{Hg}_{\mathrm{NaOH}}$ & 0.90 & 0.77 & & & & & 0.44 & 0.74 & 0.40 & 0.66 \\
\hline
\end{tabular}

Significant correlation coefficients $(p<0.05)$ (g) in Endogleyic Phaeozem (profile 6), fine-textured Endogleyic Fluvisol (profile 5) and in Haplic Luvisol (profile 4 ), the percentage of $\mathrm{Hg}_{\mathrm{DTPA}}$ forms was greater in the entire profile. This could be the result of in situ formed alkyl mercury compounds, and the migration of mobile mercury forms from higher horizons (Barnett and Turner 2001; Montgomery et al. 2000). The consequence of the occurrence of favourable alkylation conditions may be the increase in the content of especially toxic methyl and ethyl mercury, which as bioavailable forms constitute greater danger for all living organisms (Boening 2000; Han et al. 2003; Tsiros and Ambrose 1999). According to Gilmour and Henry (1991) and Paterson et al. (1990) even in such conditions, the share of alkyl mercury forms usually does not exceed $3 \%$ of the total content of this metal in soils.

\section{Mercury bound to organic matter}

According to some authors (Biester et al. 2002a; Dmytriw et al. 1995), the forms of mercury extractable by $\mathrm{NaOH}$ are mainly typical humus compounds.

The concentration of $\mathrm{Hg}_{\mathrm{NaOH}}$ in the analysed soils ranged between 0.78 and $159.37 \mu \mathrm{g} \mathrm{kg}^{-1}$, and the profile distribution was proportionally close to the distribution of total $\mathrm{Hg}$ content (Table 2). It confirms the positive significant correlation coefficient between $\mathrm{Hg}_{\text {tot }}$. and $\mathrm{Hg}_{\mathrm{NaOH}}$ $(r=0.90 ; \quad p<0.05$, Table 3$)$. Similar results were determined by Malczyk (2000) in unpolluted forest soils. This indicates that organic matter plays a dominant role in binding of mercury in soils. Mercury often forms stable complexes with organic ligands with a stability constant ranging from 18.4 to 22.1 (Stein et al. 1996).

Due to the complexity of the organic matter transformations in soil, scientific reports give contradictory information in this aspect. Wang et al. (1997) observed that the increase of humus in soils affects the decrease of $\mathrm{Hg}$ content in plants, which could indicate strong binding of this element by organic matter. Montgomery et al. (2000), however, found relatively high concentration of mobile and available mercury forms in soils with comparatively high amount of organic matter. Furthermore, the influence of soil humus on binding of mercury is dependent on the clay content, and whether clay is high may even play the dominant role in this process (Inácio et al. 1998; Wang et al. 1997).

The proved lack of $\mathrm{pH}$ influence on the content of $\mathrm{Hg}_{\mathrm{NaOH}}$ in the analysed soils (no significant correlation coefficient) may be caused by either the binding of mercury by organic matter regardless of $\mathrm{pH}$ value, or by the fact that determined $\mathrm{pH}$ range favoured such complexation. Gabriel and Williamson (2004) noticed the dominant influence of organic matter on binding mercury in soils, in which $\mathrm{pH}$ was lower than 7. This process occurred in both aerobic and anaerobic conditions. 
The percentage of $\mathrm{Hg}_{\mathrm{NaOH}}$ forms in the examined soils ranged from 2.34 to $73.70 \%$ of the total content of mercury (Table 2). Other authors indicated that in some soils the organic matter binds about $30 \%$ (Munthe et al. 2001), and even 80-85\% of total mercury content (Dmytriw et al. 1995; Henderson et al. 1998; Renneberg and Dudas 2001).

The highest percentage of $\mathrm{Hg}_{\mathrm{NaOH}}$ in the total content of mercury was determined in surface horizons of the studied soils. The share decreased with the depth in the soil profiles. Such $\mathrm{Hg}_{\mathrm{NaOH}}$ distribution was mainly connected with the content of organic carbon in profiles of soil horizon, which is confirmed by a significant positive correlation coefficient between this form of mercury and total organic carbon content ( $r=0.77 ; p<0.05$, Table 3 ). A rather low percentage content of mercury bound with organic matter in enrichment horizons (Bt) of Luvisols (profiles 1, 2 and 3) could be a result of formation of Fe-clay complexes responsible for binding mercury in these horizons, and not of greater Fe-humus-clay or humus-clay complexes (Dmytriw et al. 1995; Schlüter 1997).

In Fluvisols, the percentage of $\mathrm{Hg}_{\mathrm{NaOH}}$ in the total content of mercury in separate layers, despite its varied concentration, was relatively even. An exception was the surface horizon of Endogleyic Fluvisol (profile 5), in which the percentage of this mercury form $(2.34 \%)$ was the lowest in all of the horizons of the examined soils, showing, however, high values in the remaining subsurface horizons (40.56-48.80\%). Also, a considerably lower percentage of $\mathrm{Hg}_{\mathrm{NaOH}}$ as compared with the remaining part of the profile was determined in the deepest horizon of the Eutric Fluvisol (31.59\%), with content in the remaining part of the profile of 49.0-54.56\% (profile 4). This was most probably due to different texture of these horizons in comparison with the rest of the profile (especially varied content of clay fraction, Table 1), or the difference in humus composition (Boszke et al. 2004; Wallschläger et al. 1998b).

After statistical analysis, correlations between the content of $\mathrm{Hg}_{\mathrm{NaOH}}$ and the content of clay as well as free $\left(\mathrm{Fe}_{\mathrm{d}}\right)$ and amorphous $\left(\mathrm{Fe}_{\mathrm{o}}\right)$ ferric oxides $(r=0.40, r=0.44$, $r=0.74 ; p<0.05$, respectively, Table 3 ), has been confirmed. This may be due to the fact that during extraction procedure $(1 \mathrm{M} \mathrm{NaOH})$, the solution contained exchangeable forms of mercury, bound to these soil elements (Wang et al. 1997). Even taking this into account, their percentage in soils does not usually exceed $3 \%$ of the total content of mercury (Panyametheekul 2004). Moreover, the application of $1 \mathrm{M} \mathrm{NaOH}$ solution, in comparison with other reagents used for extraction of typical humus fractions (e.g. $\mathrm{Na}_{4} \mathrm{P}_{2} \mathrm{O}_{7}$ ), allows us to assess mercury bound to organic matter, obtaining by this way the fraction more similar to the actual content of this $\mathrm{Hg}$ form in soil (Hall and Pelchat 1997; Schnitzer and Khan 1978).
The content of $\mathrm{Hg}_{\mathrm{NaOH}}$ forms was significantly, positively correlated with cation exchange capacity $(r=0.66 ; p<0.05$, Table 3), which is linked with high sorption capacity of organic matter (Gabriel and Williamson 2004) and high affinity of $\mathrm{Hg}$ to functional groups containing sulphur (Kabata-Pendias and Pendias 2000; Skyllberg et al. 2003; Xia et al. 1998) proved in their research that from 50 to $70 \%$ of the total sulphur content in soils was included in the functional groups (mainly thiol) of organic matter, which bind metallic mercury as well as alkyl compounds very easily.

\section{Conclusions}

The availability of mercury for plants in the analysed soils was very low, on average amounting to $2.45 \%$ of the total content of this metal. It was mainly dependent on texture, the amount of organic matter and soil $\mathrm{pH}$. In the horizons enriched in both organic carbon and clay fraction, the share of water-soluble $\mathrm{Hg}$ and DTPA-extractable forms was the lowest. Considering low percentage of mobile mercury forms in total content, the risk that the metal shall migrate into deeper horizons of the soil profile and ground water is rather small. Moreover, even in soils with comparatively high total content of mercury, its availability for plants is very low. Only considerable decrease of $\mathrm{pH}$ value simultaneously with more intensive mineralization of organic matter, responsible for binding majority of mercury in the analysed soils (up to $73.7 \%$ ), could significantly affect mobility and/or toxicity of this metal.

Open Access This article is distributed under the terms of the Creative Commons Attribution 4.0 International License (http://crea tivecommons.org/licenses/by/4.0/), which permits unrestricted use, distribution, and reproduction in any medium, provided you give appropriate credit to the original author(s) and the source, provide a link to the Creative Commons license, and indicate if changes were made.

\section{References}

Alloway BJ (1995) Heavy metals in soils. 2nd edn. Springer, Netherlands

Barnett MO, Turner RR (2001) Bioaccessibility of mercury in soils. Soil Sediment Contam Int J 10(3):301-316. doi:10.1080/ 20015891109275

Biester H, Müller G, Schöler HF (2002a) Binding and mobility of mercury in soils contaminated by emissions from chlor-alkali plants. Sci Total Environ 284(1-3):191-203. doi:10.1016/ S0048-9697(01)00885-3

Biester H, Müller G, Schöler HF (2002b) Estimating distribution and retention of mercury in three different soils contaminated by emissions from chlor-alkali plants: Part I. Sci Total Environ 284(1-3):177-189. doi:10.1016/S0048-9697(01)00884-1 
Boening DW (2000) Ecological effects, transport, and fate of mercury: a general review. Chemosphere 40(12):1335-1351. doi:10.1016/S0045-6535(99)00283-0

Boszke L, Kowalski A, Głosińska G, Głosińska R, Siepak J (2003) Environmental factors affecting speciation ofmercury in the bottom sediments; an overview. Polish J Environmental Stud 12:5-13

Boszke L, Kowalski A, Siepak J (2004) Grain size partitioning of mercury in sediments of the Middle Odra River (Germany/ Poland). Water Air Soil Pollut 159(1):125-138. doi:10.1023/B: WATE.0000049171.22781.bd

Campbell L, Dixon DG, Hecky RE (2003) A review of mercury in Lake Victoria, East Africa: implications for human and ecosystem health. J Toxicol Environ Health, Part B 6(4):325-356. doi:10.1080/10937400306474

Dmytriw R, Mucci A, Lucotte M, Pichet P (1995) The partitioning of mercury in the solid components of dry and flooded forest soils and sediments from a hydroelectric reservoir, Quebec (Canada). Water Air Soil Pollut 80(1-4):1099-1103. doi:10.1007/ BF01189770

Dreher GB, Follmer LR (2004) Mercury content of Illinois soils. Water Air Soil Pollut 156(1):299-315. doi:10.1023/B:WATE. 0000036824.07207.16

Fitzgerald W (1995) Is mercury increasing in the atmosphere? The need for an atmospheric mercury network (AMNET). Water Air Soil Pollut 80(1-4):245-254. doi:10.1007/BF01189674

Gabriel M, Williamson D (2004) Principal biogeochemical factors affecting the speciation and transport of mercury through the terrestrial environment. Environ Geochem Health 26(3-4):421-434. doi:10.1007/s10653-004-1308-0

Gilmour CC, Henry EA (1991) Mercury methylation in aquatic systems affected by acid deposition. Environ Pollut 71(2-4):131-169. doi:10.1016/0269-7491(91)90031-Q

Gochfeld M (2003) Cases of mercury exposure, bioavailability, and absorption. Ecotoxicol Environ Saf 56(1):174-179. doi:10.1016/ S0147-6513(03)00060-5

Hall GEM, Pelchat P (1997) Comparison of two reagents, sodium pyrophosphate and sodium hydroxide, in the extraction of labile metal organic complexes. Water Air Soil Pollut 99(1-4):217-223. doi:10.1007/BF02406861

Han Y, Kingston HM, Boylan HM, Rahman GMM, Shah S, Richter RC, Link DD, Bhandari S (2003) Speciation of mercury in soil and sediment by selective solvent and acid extraction. Anal Bioanal Chem 375(3):428-436. doi:10.1007/s00216-002-1701-4

Henderson PJ, McMartin I, Hall GE, Percival JB, Walker DA (1998) The chemical and physical characteristics of heavy metals in humus and till in the vicinity of the base metal smelter at Flin Flon, Manitoba, Canada. Environ Geol 34(1):39-58. doi:10. 1007/s002540050255

Inácio MM, Pereira V, Pinto MS (1998) Mercury contamination in sandy soils surrounding an industrial emission source (Estarreja, Portugal). Geoderma 85(4):325-339. doi:10.1016/S00167061(98)00027-5

IUSS Working Group WRB (2014) World Reference Base for Soil Resources 2014. World Soil Resources Reports No. 106. FAO, Rome

Jacobson AR, Martínez CE, Spagnuolo M, McBride MB, Baveye P (2005) Reduction of silver solubility by humic acid and thiol ligands during acanthite $(\beta-\mathrm{Ag} 2 \mathrm{~S})$ dissolution. Environ Pollut 135(1):1-9. doi:10.1016/j.envpol.2004.10.017

Kabata-Pendias A, Pendias H (2000) Trace elements in soil and plants. 3rd edn. CRC Press, Boca Raton, Fla. London, p 413

Lindqvist $\mathrm{O}$, Johansson $\mathrm{K}$, Bringmark L, Timm B, Aastrup M, Andersson A, Hovsenius G, Håkanson L, Iverfeldt A, Meili M (1991) Mercury in the Swedish environment—recent research on causes, consequences and corrective methods. Water, Air, Soil Pollut 55(1-2):xi-261. doi:10.1007/BF00542429

Lindsay WL, Norvell WA (1978) Development of a dtpa soil test for zinc, iron, manganese, and copper1. Soil Sci Soc Am J 42(3):421-428. doi:10.2136/sssaj1978.03615995004200030009x

Malczyk P (2000) Forms of mercury in soils of selected forest ecosystems of Poland. Mengen und Spurenelemente 20:529-535

Mehra OP, Jackson ML (1960) Iron oxide removal from soil and clay by dithionite citrate system, buffered with sodium dicarbonate. Clays Clay Miner 7:317-327

Montgomery S, Lucotte M, Rheault I (2000) Temporal and spatial influences of flooding on dissolved mercury in boreal reservoirs. Sci Total Environ 260(1-3):147-157. doi:10.1016/S00489697(00)00559-3

Munthe J, Lyvén B, Parkman H, Lee Y-H, Iverfeldt Å, Haraldsson C, Verta M, Porvari P (2001) Mobility and methylation of mercury in forest soils development of an in-situ stable isotope tracer technique and initial results. Water Air Soil Pollut Focus 1(3-4):385-393. doi:10.1023/A:1017574103867

Panyametheekul S (2004) An operationally defined method to determine the speciation of mercury. Environ Geochem Health 26(1):51-57. doi:10.1023/B:EGAH.0000020967.03217.5f

Paterson S, Mackay D, Tam D, Shiu WY (1990) Uptake of organic chemicals by plants: a review of processes, correlations and models. Chemosphere 21(3):297-331. doi:10.1016/00456535(90)90002-B

Renneberg AJ, Dudas MJ (2001) Transformations of elemental mercury to inorganic and organic forms in mercury and hydrocarbon co-contaminated soils. Chemosphere 45(6-7):1103-1109. doi:10.1016/S0045-6535(01)00122-9

Różański S (2009) The content of mercury in arable soils considering pedogenic, lithogenic and anthropogenic factors. Fresenius Environ Bull 18(7):1161-1166

Sánchez DM, Quejido AJ, Fernández M, Hernández C, Schmid T, Millán R, González M, Aldea M, Martín R, Morante R (2005) Mercury and trace element fractionation in Almaden soils by application of different sequential extraction procedures. Anal Bioanal Chem 381(8):1507-1513. doi:10.1007/s00216-0053058-y

Schlüter K (1997) Sorption of inorganic mercury and monomethyl mercury in an iron-humus podzol soil of southern Norway studied by batch experiments. Environ Geol 30(3-4):266-279. doi:10.1007/s002540050156

Schlüter K (2000) Review: evaporation of mercury from soils. An integration and synthesis of current knowledge. Environ Geol 39(3-4):249-271. doi:10.1007/s002540050005

Schnitzer M, Khan SU (1978) Soil organic matter. Elsevier Scientific Publishing Company, New York

Schroeder WH, Munthe J (1998) Atmospheric mercury-an overview. Atmos Environ 32(5):809-822. doi:10.1016/S13522310(97)00293-8

Schuster E (1991) The behavior of mercury in the soil with special emphasis on complexation and adsorption processes-a review of the literature. Water Air Soil Pollut 56(1):667-680. doi:10. 1007/BF00342308

Schwertmann U (1964) Differenzierung der Eisenoxide des Bodens durch Extraktion mit Ammoniumoxalat-Lösung. Zeitschrift für Pflanzenernährung Düngung und Bodenkunde 105:194-202

Skyllberg U, Qian J, Frech W, Xia K, Bleam W (2003) Distribution of mercury, methyl mercury and organic sulphur species in soil, soil solution and stream of a boreal forest catchment. Biogeochemistry 64(1):53-76. doi:10.1023/A:1024904502633

Stein ED, Cohen Y, Winer AM (1996) Environmental distribution and transformation of mercury compounds. Crit Rev Environ Sci Technol 26(1):1-43. doi:10.1080/10643389609388485 
Tsiros IX, Ambrose RB (1999) An environmental simulation model for transport and fate of mercury in small rural catchments. Chemosphere 39(3):477-492. doi:10.1016/S00456535(98)00601-8

Tsuchiya A, Hinners TA, Burbacher TM, Faustman EM, Mariën K (2008) Mercury exposure from fish consumption within the Japanese and Korean communities. J Toxicol Environ Health, Part A 71(15):1019-1031. doi:10.1080/01932690801934612

Wallschläger D, Desai MVM, Spengler M, Wilken R-D (1998a) Mercury speciation in floodplain soils and sediments along a contaminated river transect. J Environ Qual 27(5):1034-1044. doi:10.2134/jeq1998.00472425002700050008x

Wallschläger D, Desai MVM, Spengler M, Windmöller CC, Wilken R-D (1998b) How humic substances dominate mercury geochemistry in contaminated floodplain soils and sediments. J Environ Qual 27(5):1044-1054. doi:10.2134/jeq1998. $00472425002700050009 x$

Wang DY, Qing CL, Guo TY, Guo YJ (1997) Effects of humic acid on transport and transformation of mercury in soil-plant systems. Water Air Soil Pollut 95(1-4):35-43. doi:10.1023/A: 1026468724284

Xia K, Skyllberg UL, Bleam WF, Bloom PR, Nater EA, Helmke PA (1998) X-ray absorption spectroscopic evidence for the complexation of $\mathrm{Hg}(\mathrm{II})$ by reduced sulfur in soil humic substances. Environ Sci Technol 33(2):257-261. doi:10.1021/es980433q 